\title{
Focused Ion Beam Characterization of Bicomponent Polymer Fibers
}

\author{
K.C. Wong ${ }^{1,3}$, N. Anantharamaiah ${ }^{2}$, R.R. Garcia ${ }^{1}$, A.D. Batchelor ${ }^{1,3}$, B. Pourdeyhimi ${ }^{2}$, and D.P. \\ Griffis 1,3
}

\begin{abstract}
${ }^{1}$ Analytical Instrumentation Facility, North Carolina State University, Campus Box 7531 Room 318 EGRC, 2410 Campus Shore Dr., Raleigh, NC 27695

${ }^{2}$ Nonwovens Cooperative Research Center, The Nonwovens Institute, North Carolina State University, Raleigh, NC 27695

${ }^{3}$ Department of Materials Science and Engineering, North Carolina State University, Campus Box 7907, Raleigh, NC 27695-7907
\end{abstract}

Previous work has shown that focused ion beam (FIB) can be effectively utilized for the cross-sectional analysis of polymers such as core-shell solid micro-spheres [1] and hollow latex nanospheres [2]. While these have clearly demonstrated the precise location selection and milling control provided by the FIB technique, the samples studied consisted of only a single polymer. In this work, FIB is used to investigate bicomponent polymeric fiber systems by taking advantage of the component's differing physical properties. An approach for cross sectioning and thus revealing the morphology with respect to the polymeric components in a bicomponent polymeric fiber crosssection with the island-in-the-sea (I/S) structure is presented. The I/S fibers investigated were fabricated using the spunbonding process and are composed of bicomponent combinations of polypropylene (PP), polylactic acid (PLA), polyethylene terephthalate (PET), or nylon 6 (PA6).

Bicomponent I/S fibers [3] were coated with approximately $30 \mathrm{~nm}$ of palladium-gold using a Denton Desk II sputter coater. An FEI Quanta 200 3D Dual Beam system was used for crosssectioning and imaging. The fibers were cross-sectioned by using a $30 \mathrm{keV} \mathrm{Ga}^{+}$ion beam for both the mass removal and cross-section polishing steps of the process. Initial mass removal was carried out using a water injection system to chemically enhance material removal [4]. The injection of the water vaper into the vacuum chamber resulted in an increase of chamber pressure of about 1 order of magnitude (from about 5E-6 Torr to 5E-5 Torr). Prior to the mass removal step, a platinum strip was deposited onto a selected area across the width of the fiber to protect the surface of the fiber by preventing unwanted etching of the fiber surface. An approximately $20 \times 20 \mu \mathrm{m}^{2}$ area with a depth equals to the fiber diameter $(\sim 10 \mu \mathrm{m})$ was removed in the mass removal step by using a $20 \mathrm{nA}$ beam and a rectangle raster pattern. Beam currents of $5 \mathrm{nA}, 1 \mathrm{nA}$ and $100 \mathrm{pA}$ were subsequently used for cross-section polishing using a cleaning cross-section milling pattern to provide line-by-line material removal. Finally, a 30pA beam with a dwell time of $55 \mu$ s and a $1024 \times 768$ pixels scan resolution was directed perpendicular to the cross section surface to provide a secondary electron (SE) image. An example of an I/S fiber cross-section is presented in Figure 1. Differential sputtering of the respective polymers gave rise to the necessary topographical contrast since sputtering occurred over the cross-section surface during ion induced secondary electron imaging. This differential sputtering combined with the contrast generated by ion-induced secondary electron microscopy provided the ability to differentiate the structure of the bicomponent fiber.

Various spunbonding process parameters govern the final I/S fiber structure and properties. Previous studies on revealing the cross-section morphology utilizing oxygen plasma [5-6] and scanning electron microscopy (SEM) can also provide structural information. However, the sample preparation and the necessary transfer between the different instrumentations during the examination 
process can be troublesome and there is no ability to perform site specific investigation as is provided by the FIB.

The ability to provide site specific, rapid quality control and cross-section morphology characterization for the spunbonding fabrication process of bicomponent polymeric fiber using FIB was demonstrated. With the increasing interest in polymeric materials research in recent years, differential sputtering of multi-component polymer blend using the FIB instrumentation could provide a new route for the characterization of surface and cross-sectional topography and morphology of new polymeric materials.

\section{References}

[1] L. Barner et al., J. Polymer Sci. Polymer Chem., 42 (2004) 5067.

[2] E. Beach et al., Polymer, 46 (2005) 11195.

[3] Prepared by the North Carolina State University Nonwovens Cooperative Research Center, Raleigh, NC 27695.

[4] T. Stark et al., J. Vac. Sci. Technol. B,13 (1995) 2685-2688.

[5] R. Garcia et al., Microsc. Today, 13 (2005), 38.

[6] N. Preschilla et al., Microsc. Microanal., 11(Supp 2) (2005) 2110.
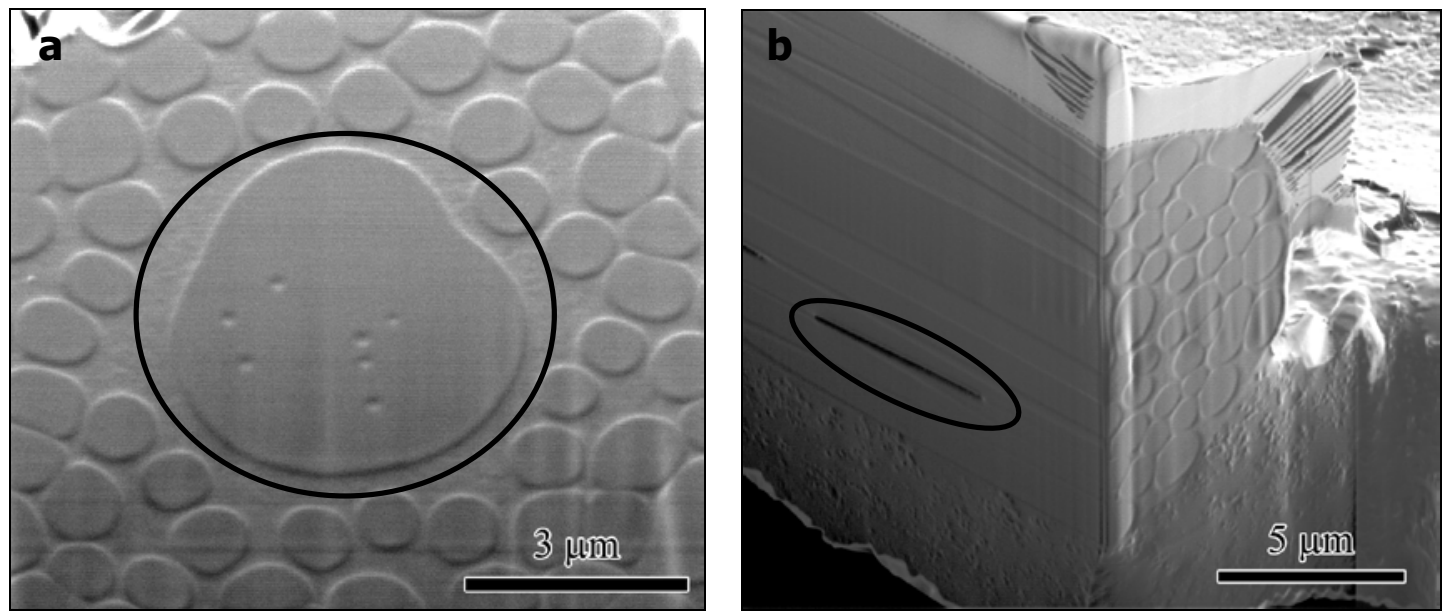

Fig. 1. Defects can be observed in the ion induced secondary electron images of an I/S fiber cross section a) end-on (island fiber agglomeration defect) and b) along the length of the fiber (void defect). This I/S fiber is composed of 108 nylon 6 (PA6) island fibers inside a polyethylene terephthalate (PET) sea.

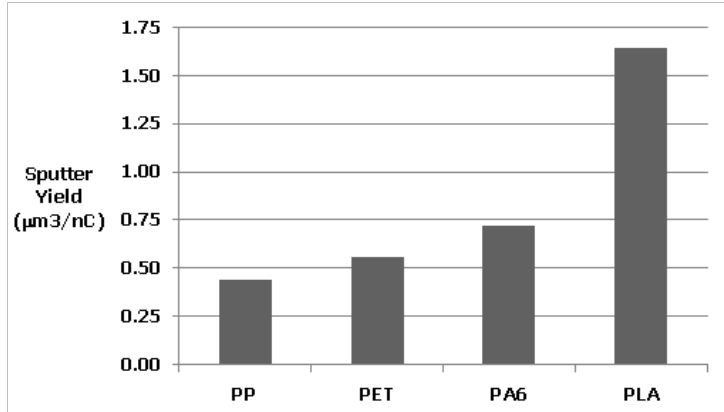

Fig. 2. The sputter yield of the polymers in this study. The sputtering conditions for all polymers were $10 \times 10 \mu \mathrm{m}^{2}$ sputtered area with a total dose of $250 \mathrm{nC}$ using a $30 \mathrm{keV} \mathrm{Ga}^{+}$. 